\title{
Economics of environmental regime shifts
}

\author{
Florian Wagener*
}

21st August 2013

\begin{abstract}
Economic consequences of critical transitions or regime shifts of ecological systems are a source of considerable concern. Though the underlying mechanisms are well-known, only since the advent of electronic computers the analysis of economic models exhibiting critical transitions can be attacked systematically. In particular bifurcation theory allows to present the qualitative effects of parameter changes in a convenient way. This article gives an overview of recent economic literature dealing with potential environmental regime shifts with a focus on the lake model.

Keywords: Regime shifts, bifurcations, environment, complex systems, catastrophes
\end{abstract}

\section{Contents}

${ }^{*}$ Center for Nonlinear Dynamics in Economics and Finance (CeNDEF), Department of Economics and Econometrics, Universiteit van Amsterdam, Valckenierstraat 65-67, 1018 XE Amsterdam, The Netherlands, F.O.O.Wagener@uva.nl 
3 Optimal management 6

3.1 Affectors and enjoyers ................. 6

3.2 Analysis of long term steady states . . . . . . . . . . . . 7

3.3 Analysis of dynamic solutions . . . . . . . . . . . . 10

3.4 Classification of solutions . . . . . . . . . . . . . . . . 14

4 Game 16

4.1 Steady state analysis . . . . . . . . . . . . . . 17

4.2 Dynamics: open-loop Nash . . . . . . . . . . . . . . 19

4.3 Dynamics: closed-loop Nash . . . . . . . . . . . . . . 19

5 Taxes $\quad 24$

5.1 Time-dependent tax rates in the lake problem . . . . . . . . 24

5.2 State-dependent taxes in the lake problem . . . . . . . . . 25

5.3 Time-dependent taxes in a global warming model . . . . . . . 25

5.4 Constant tax rates in an extended lake problem . . . . . . . . . 27

6 Uncertain regime shifts 30

7 Conclusion $\quad 34$

\section{Introduction}

Many systems can exhibit qualitatively different behaviour; which of these is selected is a function of the history of the system. This phenomenon is common in economics: we talk of societies languishing in poverty traps; evolutionary game theory explains why in a given country all cars drive on the same side of the road, though it does not make predictions about which side this will be; and archaeologists tell us that 6000 year ago, the Sahara desert was a pleasant place to stay. All of these are regimes of the system in question.

A regime is a collection of states with similar characteristics. Big external shocks can transport a system from one regime to another. More usual are accumulating processes exhibiting positive feedbacks. In economics, early docu- 
mented instances of such a feedback mechanisms are increasing returns to scale, perspicaciously described by Adam Smith in his discussion of the pin factory. This mechanism effected one of the most far-reaching regime shifts, transporting western society from the agricultural-manufactural state to the industrial state.

The overriding interest of the problem of the existence of a general equilibrium, and the related hope that this equilibrium might be stable under some general conditions, has over time fostered a huge research effort in mechanisms that ensure stability, putting emphasis on static rather than dynamic aspects of economic systems. The literature on destabilising mechanisms is comparatively much smaller, but typically in times of actual or impending economic crisis, the dynamic aspects of economies have a tendency to return to the fore.

In these days, there is ample evidence that the mean temperature of the atmosphere and the oceans is rising, and moreover that this is a consequence of human actions. This temperature rise changes the living conditions of plant and animal species, and by itself it may have serious consequences for man's economic activities.

Moreover, ecological systems may respond to an incremental increase of environmental pressure with sudden regime shifts, which have short-term and longterm economic consequences. A body of important research on the economics of ecological systems with nonconvexities has been collected in Dasgupta and Mäler (2004). This article discusses economic set-ups in which regime shifts may occur that have been developed since, techniques to analyse them, and lessons that can be learned from them. Special emphasis is put on the so-called lake or shallow lake model, as it is in a sense the simplest dynamic economic model featuring a regime shift; this occupies the first part of the article. Other approaches treat the shift to a different dynamics as occurring with a certain probability. For a recent overview of literature treating the management aspects of regime shifts, we refer the reader to Crépin et al. (2012). 


\section{Deterministic regime shifts: the lake model}

The 'lake' or 'shallow lake' model was introduced to analyse the tragedy of the commons in the situation of a lake polluted by agricultural waste. Its simplicity makes it a prototypical study object for the ramifications of optimal management decisions when dealing with a system that features positive feedback.

Lakes host intricate ecosystems; for the present purposes, an oversimplified description is sufficient, but the real object is much more complicated (Scheffer, 1998).

The bottom of a lake is formed by the sediment; the root systems of water plants hold it in place. In a clear, 'oligotrophic' state, the sunlight, which these plants need to live, filters through the water column above them. If artificial fertilisers are used on the fields around the lake, rainfall washes some of the phosphorus they contain into the lake. There it increases phytoplankton biomass in the water column as well as the periphyton layers on the water plants. Both deprive the plants of light.

When water plants die, they release the lake sediment, and the phosphorus contained in the sediment. This initiates a positive feedback loop, as the resuspended phosphorus increases the phytoplankton biomass in turn: the lake becomes turbid or 'eutrophic'. Depending on the characteristics of the lake, a return to the oligotrophic state, if at all possible, necessitates a large reduction of inflow of phosphorus.

Denote by $x=x(t)$ the amount of phosphorus suspended in the water column of the lake, by $u=u(t)$ the inflow, per unit time, of phosphorus resulting from agricultural activities, and by $b$ the sedimentation and outflow rate of phosphorus out of the water column. The following differential equation provides a model for the phosphorus concentration $x$ in the lake (Mäler et al., 2003):

$$
\dot{x}=u-g(x) .
$$


In particular, the natural dynamics $g$ of the lake is often taken to be of the form

$$
g(x)=b x-\frac{x^{2}}{x^{2}+1} .
$$

Figure 1 illustrates the resulting dynamics for constant loadings $u$ and three different values of the sedimentation rate $b$. The arrows indicate the direction of the

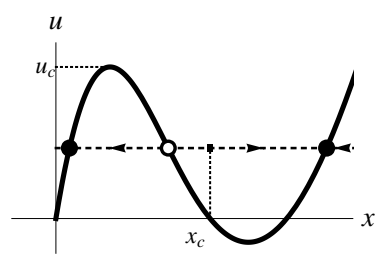

(a) Fragile lake

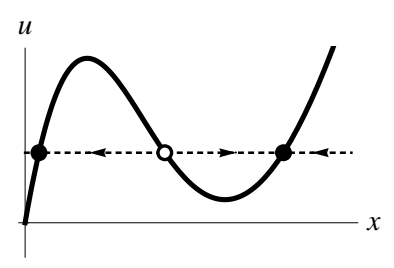

(b) Reversible lake



(c) Robust lake

Figure 1: The lake dynamics for a fragile lake $(b=0.49)$, a reversible lake $(b=0.51)$ and a robust lake $(b=0.66)$. The black square indicates the point of no return of the fragile lake.

dynamics. Taking $u$ for the moment to be a constant system parameter, it appears from Figure 1 that for some combinations $(b, u)$ the lake dynamics (1) has a single steady state, whereas for others there are three steady states. Figure 2 indicates the precise parameter regions. Increasing the value of the parameter $u$ in, for instance, Figure 1(a) destabilises the oligotrophic (left) steady state at a critical value $u_{c}$, and the system shifts to the eutrophic (right) steady state. Decreasing the value of $u$ slowly, will not shift the system back. A fragile lake cannot be restored to an oligotrophic situation at all: if the state $x(t)$ reaches the level $x_{c}$ (see Figure 1(a) for the location of $x_{c}$ ) for some time $t=t^{\prime}$, it cannot decrease past $x_{c}$ for any future time $t>t^{\prime}$ again: the regime shift is irreversible. But even if the regime shift is reversible, as in Figure 1(b), the phosphorus inflow has to be decreased to much lower levels than $u_{c}$ before the reverse regime shift occurs.

Equation (1), with $x$ as a negative capital and $u$ as a negative investment, has similar properties to capital dynamics with increasing returns to scale, that is, with non-concave production functions, which have been considered in the literature on optimal growth since the late 1960s (see e.g. Dechert and Nishimura, 1983; Krugman, 1991; Majumdar and Mitra, 1982; Romer, 1986; Sethi, 1977; Skiba, 


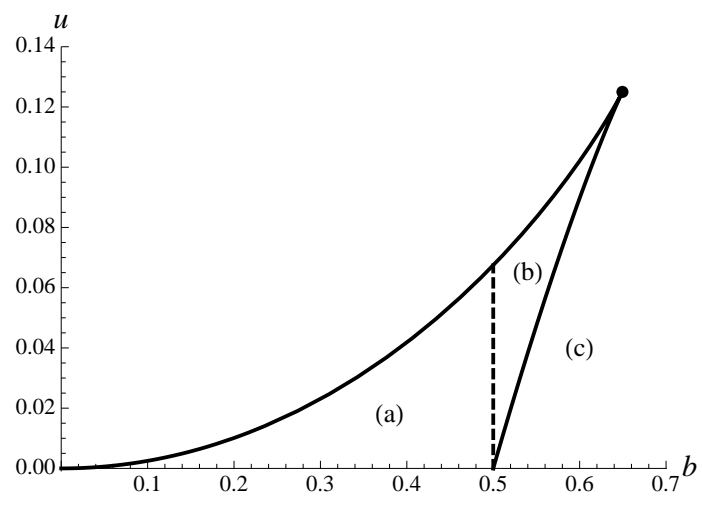

Figure 2: Bifurcation diagram for the lake dynamics (1). The labels refer back to the three typical situations depicted in Figure 1: region (a) corresponds to a fragile lake with three steady states, region (b) to a reversible lake with three steady states, and region (c) to a robust lake with only a single steady state. Two saddle-node bifurcation curves (solid), coalescing in a cusp point, bound the union of the regions ( $a$ ) and $(b)$ where there are three steady states. The line $b=\frac{1}{2}$ (dashed) divides these two regions.

1978; Treadway, 1969). Pollution models with nonconcavities were studied by Tahvonen and Salo (1996) and Brock and Starrett (2003).

\section{Optimal management}

\subsection{Affectors and enjoyers}

Equation (1) describes the ecological dynamics of the lake. An economic component enters if there are agents that use the lake. This may be direct use, by fishermen for fishing, by tourists for recreation, by a water company for freshwater, or indirect use, by farmers that use artificial fertiliser. In the terminology of Brock and Starrett (2003), the former agents are enjoyers of the lake, while the latter are affectors. The shallow lake literature assumes that the social stream of benefits $\beta_{\mathrm{s}}$ is of the form

$$
\beta_{\mathrm{s}}(x, u)=\beta_{\mathrm{a}}(u)+c \beta_{\mathrm{e}}(x) .
$$


The benefit stream $\beta_{\mathrm{a}}(u)$ of the affectors is increasing and strictly concave in the use $u$ of phosphorus, whereas the benefit stream $\beta_{\mathrm{e}}(x)$ of the enjoyers is decreasing and strictly concave in the amount of phosphorus $x$ in the water column of the lake. The parameter $c$ is a weighting parameter, expressing the relative economic importance of the enjoyers relative to the affectors of the lake.

In Mäler et al. (2003), where the economic lake model was introduced, the following specific choices were made:

$$
\beta_{\mathrm{a}}(u)=\log u, \quad \beta_{\mathrm{e}}(x)=-x^{2} .
$$

In the shallow lake optimal control problem, a manager maximises the integral $I$ of the discounted stream of benefits over an infinite time horizon

$$
I=\int_{0}^{\infty} \mathrm{e}^{-\rho t} \beta_{\mathrm{s}}(x, u) \mathrm{d} t=\int_{0}^{\infty} \mathrm{e}^{-\rho t}\left(\beta_{\mathrm{a}}(u)+c \beta_{\mathrm{e}}(x)\right) \mathrm{d} t,
$$

subject to the dynamic constraint (1).

\subsection{Analysis of long term steady states}

The lake problem almost always reduces to a quasi-static problem if future benefits are not discounted. To make this statement precise, the concept of an optimal solution of the problem has to be specified, as the integral (5) usually diverges if $\rho=0$. Rather than introducing notions like catching up or overtaking optimality (von Weizsäcker, 1965), the much simpler notion of average benefit stream is used here.

Define the finite-horizon average benefit stream

$$
A_{T}=\frac{1}{T} \int_{0}^{T} \beta_{\mathrm{s}}(x, u) \mathrm{d} t
$$

which compares the integrated undiscounted benefit stream with a constant benefit stream. The infinite-horizon average benefit stream is then

$$
A=\lim _{T \rightarrow \infty} A_{T}
$$


For trajectories tending to a steady state, the value of $A$ reduces to the value of $\beta_{\mathrm{s}}$ at the steady state, as the details of the transient dynamics do not influence the value of the limit. Only if there are several steady states with equal values of $A$, a more precise optimality criterion, like catching up or overtaking, is relevant. In the present context, this however constitutes a non-generic 'hairline' case.

In the situation without discounting, a manager has to maximise the benefit stream

$$
\beta_{\mathrm{s}}(x, u)=\beta_{\mathrm{a}}(u)+\beta_{\mathrm{e}}(x),
$$

subject to the steady state condition

$$
u-g(x)=0
$$

(compare Mäler et al., 2003, Section 3). Substitution of the latter equation into the former yields the benefit stream as a function of the state

$$
\beta(x)=\beta_{\mathrm{a}}(g(x))+\beta_{\mathrm{e}}(x) .
$$

If this is maximal, then

$$
\beta_{\mathrm{e}}^{\prime}(x)+\beta_{\mathrm{a}}^{\prime}(g(x)) g^{\prime}(x)=0 .
$$

That is, the sum of the marginal benefits which the enjoyers and the affectors derive from the lake is zero.

For the specification (2) of the lake dynamics and (4) of the benefit streams, Figure 3 shows the graph of $\beta$.

It appears that the function $\beta$ can have several local maxima. To find the parameter values for which one of these, say the left local maximum, is global, it suffices to determine those parameter values which are in the boundary of this set; these correspond to the bifurcating cases. For the situation that there are two local maxima, and the left one is global, there are two bifurcations: either the right local maximum is about to disappear in a degenerate critical point, or the two local maxima are both global. The numerical condition for the first case is 


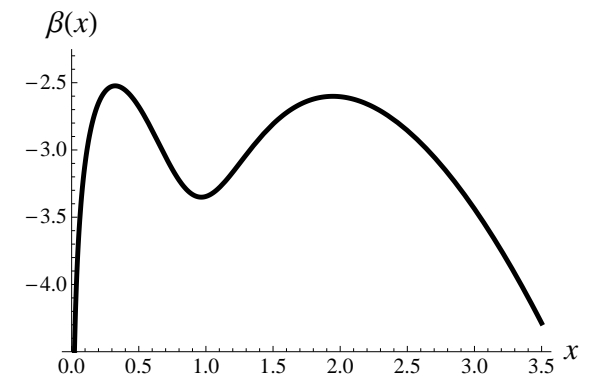

Figure 3: Total benefit stream in steady state: $b=0.55, c=0.35$.

that there are two points $x_{1}<x_{2}$, such that

$$
\begin{array}{ll}
\beta^{\prime}\left(x_{1}\right)=0, & \beta^{\prime \prime}\left(x_{1}\right)<0, \\
\beta^{\prime}\left(x_{2}\right)=\beta^{\prime \prime}\left(x_{2}\right)=0, & \beta^{\prime \prime \prime}\left(x_{2}\right) \neq 0,
\end{array}
$$

and for the second

$$
\begin{aligned}
\beta^{\prime}\left(x_{1}\right) & =\beta^{\prime}\left(x_{2}\right)=0, \quad \beta^{\prime \prime}\left(x_{1}\right)<0, \quad \beta^{\prime \prime}\left(x_{2}\right)<0, \\
\beta\left(x_{1}\right) & =\beta\left(x_{2}\right) .
\end{aligned}
$$

Figure 4 depicts the curves in the $(b, c)$-parameter plane determined by these conditions, as well as analogous conditions for the case that the right local maximum is global.

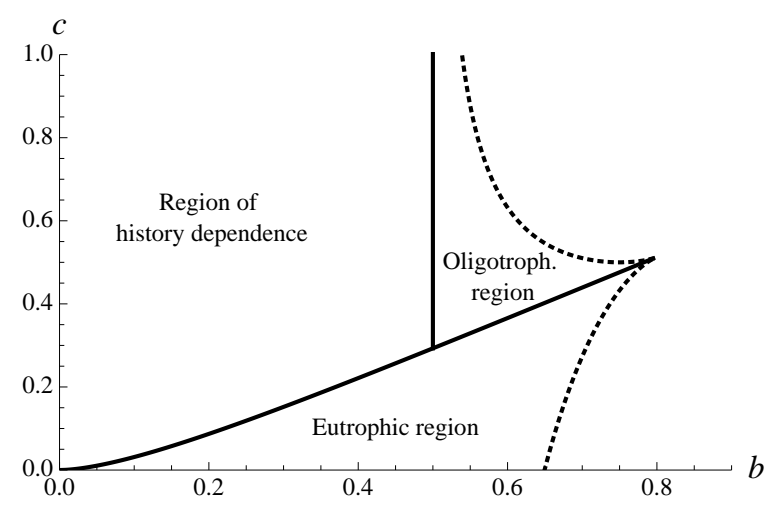

Figure 4: Bifurcation diagram of the quasi-static optimally managed lake.

There is a peculiarity in this figure, related to the line $b=\frac{1}{2}$. Recall that 
if $b \leq \frac{1}{2}$, the lake is irreversible, once it has reached an eutrophic state. The region where the oligotrophic steady state is optimal has therefore to be divided into two subregions, according to whether the oligotrophic maximum can be realised from all initial states, or whether it can be realised only from sufficiently unpolluted initial states.

Note that the interval of eutrophic $c$-values increases as $b$ increases: this reflects the fact that for robust lakes, the eutrophic states are less damaging than for fragile lakes, and therefore it is less imperative to avoid them. Optimal management gives the highest priority to conserve the most fragile ecosystems.

\subsection{Analysis of dynamic solutions}

For positive discount rates, the details of the transient dynamics are not negligible any more. Solutions to the optimal management problem are computed using the Pontryagin maximum principle (see e.g. Seierstad and Sydsaeter, 1987). For this, introduce the (current-value) Pontryagin function

$$
P(x, y, u)=\beta_{\mathrm{a}}(u)+\beta_{\mathrm{e}}(x)+y(u-g(x)) ;
$$

here $y$ is the shadow cost of pollution. The function $P$ is often called the (currentvalue) Hamilton function or the unmaximised Hamilton function. The maximum principle requires that for given $x$ and $y$, the action $u$ maximises the value of $P$.

Let $u=u^{*}(y)=\left(\beta_{\mathrm{a}}^{\prime}\right)^{-1}(-y)$ be this maximiser. The current-value Hamilton function of the problem, also called the maximised current-value Hamilton function, is then

$$
H(x, y)=\beta_{\mathrm{a}}\left(u^{*}(y)\right)+\beta_{\mathrm{e}}(x)+y\left(u^{*}(y)-g(x)\right) .
$$

The maximum principle then further requires $(x, y)=(x(t), y(t))$ to satisfy the 
system of differential equations

$$
\begin{aligned}
& \dot{x}=\quad \frac{\partial H}{\partial y}(x, y)=u^{*}(y)-g(x), \\
& \dot{y}=\rho y-\frac{\partial H}{\partial x}(x, y)=\rho y-\beta_{\mathrm{e}}^{\prime}(x)+y g^{\prime}(x),
\end{aligned}
$$

together with two additional boundary conditions in the time domain. The first of these

$$
x(0)=x_{0}
$$

just expresses that at $t=0$, the state trajectory is at the initial state $x_{0}$. The second is the transversality condition, which requires that

$$
\lim _{t \rightarrow \infty} \mathrm{e}^{-\rho t} y(t)=0
$$

if the state trajectory is eventually bounded away from the state boundary point $x=0$; that is, if there is some $\delta>0$ and some $T>0$ such that $x(t)>\delta$ for all $t>T$. If the state trajectory is not eventually bounded away from the state boundary, then the transversality condition requires that

$$
\limsup _{t \rightarrow \infty} \mathrm{e}^{-\rho t} y(t) \leq 0
$$

Equations (7), (8), (9) and (10) or (11) constitute necessary conditions for any optimal solution. These conditions take the form of a boundary value problem of a system of differential equations. The typical outcome of the maximum principle is a diagram as shown in Figure 5.

The figure shows two curves in the $(x, u)$-plane that have the property that the graph of the optimal policy function, denoted by a thick line in the figure, is necessarily a part of the union of these curves. The two curves are the union of the orbits that approach the saddle equilibria of the state-costate system, indicated by dots in the figure, as $t$ tends to infinity. The curves do not fully specify the optimal policy function, as there is a region, roughly between $x=0.6$ and $x=1.1$, where the graph of the optimal policy function could coincide with either of the curves. 


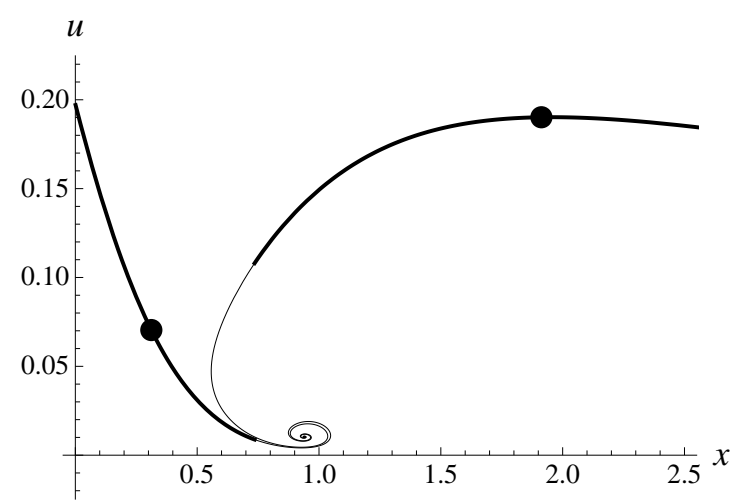

Figure 5: Candidate solutions found with the maximum principle. Parameters: $b=0.51, c=0.5, \rho=0.03$.

To resolve this ambiguity, the value of the integral $I$ has to be computed on all points of the two curves in the overlapping interval; this is usually done using numerical methods. It can be shown (Wagener, 2003) that there is exactly one point $x_{\mathrm{i}}$ in the ambiguous interval such that for all $x \leq x_{\mathrm{i}}$, the curve through the left saddle point coincides with the graph of the optimal policy function, while for $x \geq x_{\mathrm{i}}$, the same holds for the curve through the right saddle point. At $x_{\mathrm{i}}$, the policy maker is indifferent between the two branches of the policy function; the point is therefore called an indifference threshold. The reader should note that there are many names used in the literature for this concept: e.g. tie point, shock point, Maxwell point, Skiba point, Dechert-Nishimura(-Sethi)-Skiba point.

The result of the analysis is the optimal policy function, illustrated in Figure 6 .

In the figure the dashed line indicates the locus of the stabilising levels of $u$; those are the levels of $u$ which stabilise $x$ at the given value. For the left steady state $x_{\mathrm{o}}$, the optimal pollution policy is above the stabilising level if $0 \leq x<x_{\mathrm{o}}$, while it is below that level if $x_{\mathrm{o}}<x \leq x_{\mathrm{i}}$. This pushes the system towards $x_{\mathrm{o}}$ for all initial states below the indifference threshold - arrows on the horizontal axis indicate the dynamics under the optimal policy. Analogously, for all initial states above $x_{\mathrm{i}}$, the optimal policy pushes the state to the right steady state $x_{\mathrm{e}}$.

Note also the gradient of the optimal policy function: at the oligotrophic steady state, it is strongly negative, implementing a strong negative feedback 


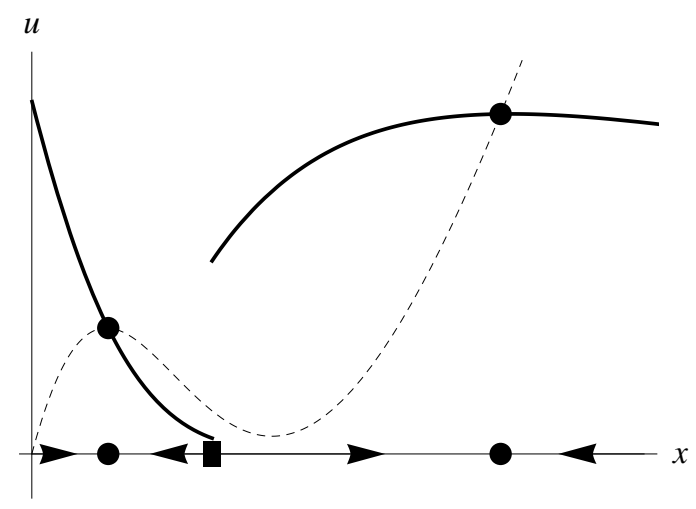

Figure 6: Optimal policy function and optimal dynamics. Intersections of the graph of the optimal policy function (solid) and the $\dot{x}=0$ isocline (dashed) give the steady states under optimal management (black circles). The resulting dynamics under optimal policy is indicated on the horizontal axis: the circles indicate the stable steady states, whereas the square indicates the indifference threshold. Same parameters as in Figure 5.

that stabilises the lake at the tipping point, whereas at the eutrophic steady state the policy function is almost constant and the natural dynamics of the lake effect its stabilisation.

The shallow lake problem depends on two additional parameters, the weight parameter $c$ introduced above, and the discount rate $\rho$, which determines the relative weight of future benefits relative to present benefits. Depending on the values of the parameters $b, c$ and $\rho$, there are three structurally stable qualitatively different types of the dynamics of the lake under optimal policy.

In this context, structural stability of a type means that by slightly changing the problem, the type of the dynamics under optimal management of the changed problem is the same as of the original problem. In a parameter diagram, a structurally stable type corresponds therefore to an open set of parameter values, as small parameter changes cannot change the type of the dynamics. The structurally stable types are the 'typical' configurations of the system dynamics.

Figure 6 shows a typical configuration: two attracting long-term steady states, separated by an indifference point. Figure 7 gives the other two: a single, globally attracting steady state, and two attracting steady states separated by a repelling steady state. In the latter configuration, the optimal values of $u$ are close to the 
stabilising values of $u$, for which $\dot{x}=0$; this implies that for that configuration, the state $x(t)$ is changing only slowly over time.

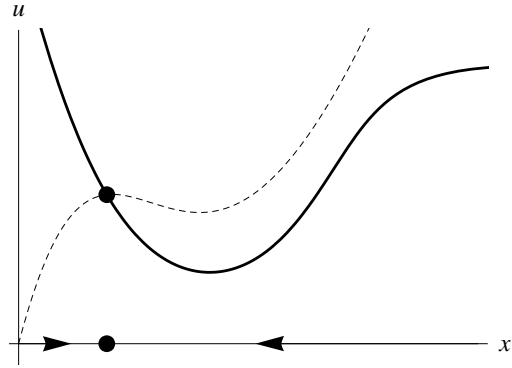

(a) Single steady state

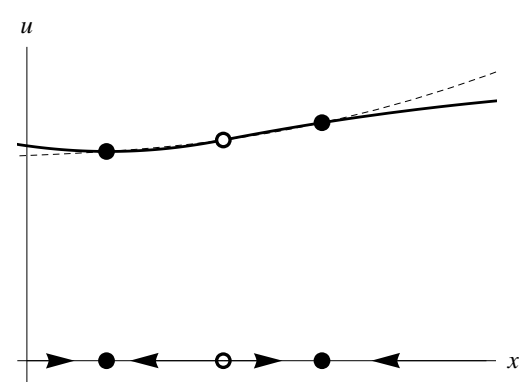

(b) Repelling threshold (detail)

Figure 7: Two types of typical state dynamics under optimal management. Left: globally asymptotically stable attracting steady state. Parameters: $b=$ 0.6, $c=0.6, \rho=0.03$. Right: two attracting steady states, separated by a repelling steady state. Parameters: $b=0.675, c=0.92, \rho=0.16$. The open circle indicates a repeller; other symbols are as in Figure 6.

\subsection{Classification of solutions}

Systems that are not structurally stable are called bifurcating. Determining parameter values of bifurcating systems consequently yields the boundaries of the parameter regions that correspond to the various structurally stable types. Kiseleva and Wagener (2011) give a classification of the possible bifurcating systems for optimal control problems with one-dimensional state spaces.

The so-called codimension expresses the relative importance of a bifurcation. The main bifurcations are the bifurcations of codimension one: the parameter sets corresponding to systems at these bifurcations are composed of unions of manifolds whose dimensions are one less than the dimension of the parameter space. Higher codimensions are defined similarly. For instance, if the parameter space is two-dimensional, codimension one bifurcations trace out onedimensional curves, codimension two bifurcations correspond to isolated points, and codimension three bifurcations do usually not occur in a two-parameter diagram. 
There are three types of codimension one bifurcations for the dynamics under optimal management: a saddle-node (SN) bifurcation, where a repeller and an attracting steady state are created or destroyed; an indifference-attractor (IA) bifurcation, where an indifference threshold and an attracting steady state are created or destroyed, and an indifference-repeller (IR) bifurcation, where an indifference threshold turns in to a repeller or vice versa. For the shallow lake model, Figure 8 illustrates the regions of structural stability, as well as the codimension one bifurcation curves, for the $(b, c)$-parameter plane with $\rho=0.03$, and for the $(c, \rho)$-parameter plane given by $b=0.65$.

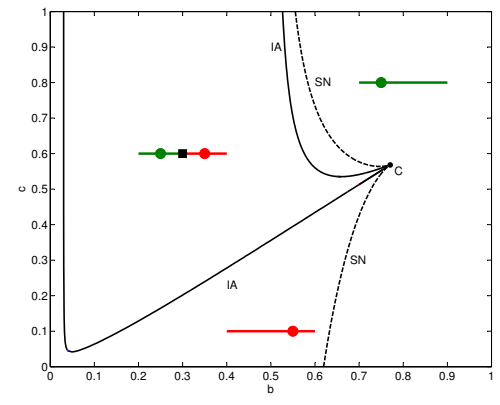

(a) $(b, c)$-bifurcation diagram

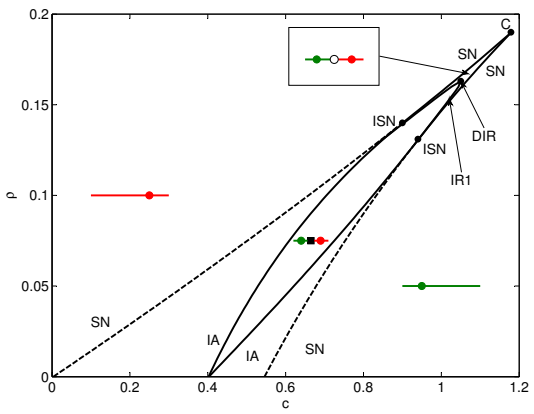

(b) $(c, \rho)$-bifurcation diagram

Figure 8: Bifurcation diagrams. Left the $(b, c)$-diagram for $\rho=0.03$; right the $(c, \rho)$-diagram for $b=0.65$. Solid curves border regions of structural stable dynamics under optimal management. Dashed curves correspond to bifurcations of the state-costate system that do not correspond to bifurcations of the state dynamics under optimal management. The abbreviations ISN, DIR, $C$ refer to codimension two bifurcation points not discussed in the text. After Kiseleva and Wagener (2010).

In the lake model, the parameter $b$ is like a technology parameter: it is a typical physical feature of a given lake. In contrast to this, the parameters $c$ and $\rho$ describe economic preferences. Figure $8(\mathrm{~b})$ is interesting, as it shows the dependency of the lake dynamics on the preferences of the decision maker. In particular, note that increasing $\rho$ always eventually leads to the lake eutrophicating. 


\section{Game}

Mäler et al. (2003) also considered a noncooperative game associated to the shallow lake system. In this game, a number of decision makers, say $n$, where $n \geq 2$, use the lake. An example would be communes or states bordering the lake. Decision maker, or player $i$ derives benefits from agricultural activities, causing a phosphorus inflow $u_{i}=u_{i}(t)$ into the lake. The amount of phosphorus in the lake is then described by

$$
\dot{x}=\sum_{i=1}^{n} u_{i}-g(x) .
$$

All players suffer from the pollution in the lake; that is, the benefits of player $i$ are given by the integral

$$
I_{i}=\int_{0}^{\infty} \mathrm{e}^{-\rho t} \beta_{\mathrm{s}, i}\left(x, u_{i}\right) \mathrm{d} t=\int_{0}^{\infty} \mathrm{e}^{-\rho t}\left(\beta_{\mathrm{a}, i}\left(u_{i}\right)+\beta_{\mathrm{e}, i}(x)\right) \mathrm{d} t .
$$

An action schedule that determines at each point in the game the pollution amount $u_{i}$ of player $i$ is called the strategy of player $i$. Strategies that consist of actions that are only conditioned on time, that is, for which $u_{i}=u_{i}(t)$, are said to be of 'open-loop' type. Other types of strategies are considered below.

The optimal pollution rate of player $i$ will depend, through the lake dynamics (12), on the choices of

$$
u_{-i}=u_{-i}(t)=\left(u_{1}(t), \cdots, u_{i-1}(t), u_{i+1}(t), \cdots, u_{n}(t)\right)
$$

of the other players. The strategies $u_{j}, j=1, \ldots, n$ form a Nash equilibrium if player $i$ 's strategy is optimal given the strategies of the other players.

For the specifications (4), Mäler et al. (2003) have investigated symmetric open-loop Nash equilibrium strategies in a game with $n$ players; that is, in equilibrium, each player uses the same strategy $u_{i}(t)=u_{\mathrm{nc}}(t)$ ('nc' for noncooperative). 


\subsection{Steady state analysis}

As for the optimal management case, a steady state analysis can be performed. Again this corresponds, except for hairline cases, to the dynamic analysis of the situation for $\rho=0$, that is, for vanishing discount rates. For the sake of simplicity, only the two-player situation $n=2$ is considered.

Given that player 2 plays the time-constant strategy $u_{2}$, player 1 maximises

$$
\beta_{1}\left(u_{1}\right)=\beta_{\mathrm{a}, 1}\left(u_{1}\right)+\beta_{\mathrm{e}, 1}(x),
$$

subject to the condition

$$
u_{1}+u_{2}-g(x)=0 \text {. }
$$

Eliminating $u_{1}$, the benefit stream $\beta_{1}$ as function of the steady state $x$ takes the form

$$
\beta_{1}(x)=\beta_{\mathrm{a}, 1}\left(g(x)-u_{2}\right)+\beta_{\mathrm{e}, 1}(x) .
$$

The condition for a maximising steady state reads as

$$
0=\beta_{\mathrm{a}, 1}^{\prime}\left(g(x)-u_{2}\right) g^{\prime}(x)+\beta_{\mathrm{e}, 1}^{\prime}(x)
$$

and it has the same interpretation as before.

The symmetry condition requires that $u_{1}=u_{2}$; if the lake is to be in steady state, then

$$
u_{1}=u_{2}=\frac{1}{2} g(x)
$$

leading to the eventual condition that

$$
0=\beta_{\mathrm{a}, 1}^{\prime}(g(x) / 2) g^{\prime}(x)+\beta_{\mathrm{e}, 1}^{\prime}(x) .
$$

As usual, this condition is necessary for a Nash equilibrium, but not sufficient. For, let $x=x^{*}$ be a state that satisfies (13); the implied actions of the players are then

$$
u_{1}^{*}=u_{2}^{*}=\frac{1}{2} g\left(x^{*}\right)
$$


The pair $\left(u_{1}, u_{2}\right)=\left(u_{1}^{*}, u_{2}^{*}\right)$ only defines a Nash strategy equilibrium if $x=x^{*}$ is a maximiser of the benefit stream of player 1 with $u_{2}=u_{2}^{*}$. There are situations where that is not true.

Identifying the bifurcations in an analogous manner as in the optimal management problem, Figure 9 shows the regions corresponding to typical situations.

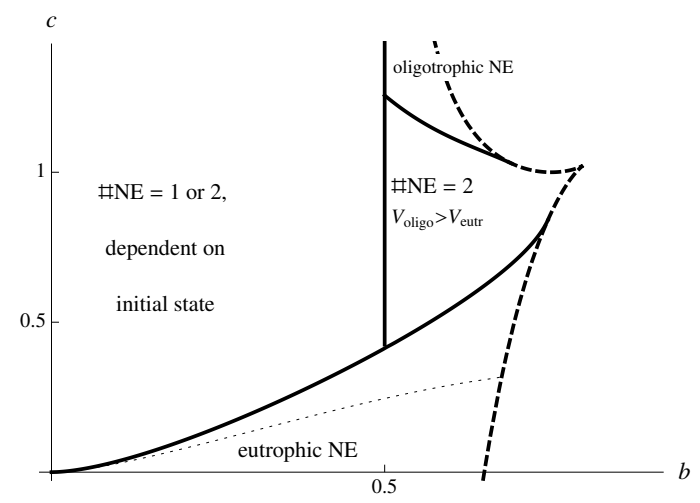

Figure 9: Steady state Nash equilibria of the lake pollution game.

The dashed curve on the right bounds the region for which there is a unique solution of equation (13), which gives the Nash equilibrium steady state, from the region for which there are three solutions, two of which, corresponding to local maxima of $\beta_{i}(x)$, are candidate Nash equilibria. Both correspond to a Nash equilibrium in the regions marked ' $\# \mathrm{NE}=2$ ' and ' $\mathrm{NE}=1$ or $=2$ '; in the latter region, the oligotrophic Nash equilibrium may not be reachable due to irreversibility of the lake dynamics, if the initial steady state of the lake is too far in the eutrophic region. In the regions marked 'oligotrophic NE' and 'eutrophic NE', only one of the two local maxima of $\beta_{i}(x)$ corresponds to a Nash equilibrium, the other being not stable under non-symmetric deviations.

Computing the payoffs $V_{\text {oligo }}$ and $V_{\text {eutr }}$ at the candidate Nash equilibria, it turns out that these are higher in the oligotrophic candidate whenever $(b, c)$ is above the dotted curve in the region marked 'eutrophic NE'. That means that in the intersection of the region where $V_{\text {oligo }}>V_{\text {eutr }}$ with the 'eutrophic NE' region, the game has the structure of the prisoner's dilemma, whereas in the region ' $\# \mathrm{NE}=2$ ', it is a stag-hunt game. 


\subsection{Dynamics: open-loop Nash}

Mäler et al. (2003) show that symmetric open-loop Nash equilibrium strategies $u_{i}(t)=u_{\mathrm{nc}}(t), i=1, \ldots, n$ of the $n$-player game with parameters $(b, c, \rho)$ also are maximisers of the optimal management problem with parameters $(b, c / n, \rho)$. It follows that if $n$ is sufficiently large, the lake is always allowed to eutrophicate. In contrast to this, the symmetric cooperative strategies $u_{i}(t)=u_{\mathrm{c}}(t)$, $i=1, \ldots, n$, have the property that $n u_{\mathrm{c}}(t)$ is a maximiser of the optimal management problem with parameters $(b, c, \rho)$. That is, if there are too many players, the lake eutrophicates, while the optimal cooperative solution would be to conserve the lake in an oligotrophic state. Put differently, the lake problem is another instance of Hardin's tragedy of the commons (Hardin, 1968).

It does however not necessarily follow that the bifurcation diagram in Figure 8(a), with $c$ replaced by $c / n$, gives the structure of the open-loop Nash equilibria. As showed in Subsection 4.1, for $\rho=0$ some candidate Nash equilibrium strategies may be unstable under non-symmetric deviations. A bifurcation diagram for $\rho>0$ where the possibility of unsymmetric deviations is taken into account has not yet been given in the literature.

\subsection{Dynamics: closed-loop Nash}

In contrast to open-loop strategies, closed-loop strategies condition actions on time as well as on the state of the system. That is $u_{i}=u_{i}(t, x)$. A subclass of closed-loop strategies are the feedback strategies, where the actions are exclusively conditioned on the state: $u_{i}=u_{i}(x)$. In infinite horizon games with exponential discounting, the optimisation problem is essentially time-invariant, and closed-loop strategies reduce to feedback strategies.

Kossioris et al. (2008) and Dockner and Wagener (2013) have found symmetric feedback strategies for the lake game numerically. To sketch the method, assume that feedback strategies $u_{-i}(x)$ of all players except player $i$ are given. Introduce 
the value function $V_{i}$ of player $i$ as

$$
V_{i}\left(x_{0}\right)=\sup \int_{0}^{\infty} \mathrm{e}^{-\rho t}\left(\beta_{\mathrm{a}, i}\left(u_{i}\right)+\beta_{\mathrm{e}, i}(x)\right) \mathrm{d} t
$$

where the supremum is taken over all pollution schedules $u_{i}$, subject to the lake dynamics (12) as well as the initial condition $x(0)=x_{0}$. The Pontryagin function of player $i$ reads as

$$
P_{i}\left(x, y_{i}, u_{i}\right)=\beta_{\mathrm{a}, i}\left(u_{i}\right)+\beta_{\mathrm{e}, i}(x)+y_{i}\left(u_{i}+\sum_{j \neq i} u_{j}(x)-g(x)\right) .
$$

Then the value function $V_{i}$ satisfies the Hamilton-Jacobi-Bellman equation

$$
\rho V_{i}(x)=\max _{u_{i}} P\left(x, V_{i}^{\prime}(x), u_{i} ; u_{-i}(x)\right) .
$$

It can be shown that $V_{i}$ is continuous for all $x$; at points where the value function is non-differentiable, the Hamilton-Jacobi-Bellman equation is satisfied in the sense of viscosity solutions. In the present context, points of non-differentiability are generically isolated. The viscosity solution prescribes precisely in which way $V_{i}^{\prime}$ can jump at a point of non-differentiability between the values of $V_{i}^{\prime}$ that yield the same value $\rho V_{i}(x)$ of the right hand side of (14). In practice, these are the natural jump conditions.

Analogously to the optimal management case, the maximisation in (14) yields a relation

$$
u_{i}=u_{i}^{*}\left(V_{i}^{\prime}(x)\right)
$$

where $u_{i}^{*}\left(y_{i}\right)=\left(\beta_{\mathrm{a}, i}^{\prime}\right)^{-1}\left(-y_{i}\right)$. These relations hold for every $i=1, \cdots, n$. Substitution back into (14) yields

$$
\rho V_{i}=H_{i}\left(x, V_{1}^{\prime}(x), \cdots, V_{n}^{\prime}(x)\right),
$$


where

$$
H_{i}\left(x, y_{1}, \cdots, y_{n}\right)=\beta_{\mathrm{a}, i}\left(u_{i}^{*}\left(y_{i}\right)\right)+\beta_{\mathrm{e}, i}(x)+y_{i}\left(\sum_{j \neq i} u_{j}^{*}\left(y_{j}\right)-g(x)\right)
$$

is the Hamilton function of player $i$ in the game. Taking equation (15) repeatedly for $i=1, \cdots, n$ yields the system of Hamilton-Jacobi equations for the value functions of the players in a Nash equilibrium of feedback strategies.

In the symmetric situation, where the benefit streams are equal for all players, it is possible that the feedback strategies, the Hamilton functions and the associated value functions are also the same for all players. Then the system of Hamilton-Jacobi equations reduces to the single equation

$$
\rho V=H_{\text {symm }}\left(x, V^{\prime}(x)\right)
$$

with

$$
H_{\text {symm }}(x, y)=\beta_{\mathrm{a}}\left(u^{*}(y)\right)+\beta_{\mathrm{e}}(x)+y\left((n-1) u^{*}(y)-g(x)\right) .
$$

For the specifications (2) and (4) of the lake problem, equation (17) reads as

$$
\rho V(x)=-\log \left(-V^{\prime}(x)\right)-c x^{2}-V^{\prime}(x) g(x)-(n-1) .
$$

Equation (17) is an implicit differential equation for $V$; there is no initial condition. To solve the equation, introduce $y(x)=V^{\prime}(x)$, differentiate both sides once with respect to $x$, and rearrange terms to obtain

$$
\frac{\partial H_{\text {symm }}}{\partial y}(x, y(x)) y^{\prime}(x)=\rho y(x)-\frac{\partial H_{\text {symm }}}{\partial x}(x, y(x)) .
$$

This is sometimes called the shadow price equation (see Case, 1979; Dockner and Van Long, 1994; Rincón-Zapatero et al., 1998; Tsutsui and Mino, 1990; Wirl, 1996). Dockner and Wagener analyse this equation by remarking that a curve $(x(s), y(s))$ traces out the graph of $y=y(x)$ around a point where $y(x)$ is dif- 
ferentiable, if

$$
x^{\prime}(s)=\frac{\partial H_{\text {symm }}}{\partial y}(x(s), y(s)), \quad y^{\prime}(s)=\rho y-\frac{\partial H_{\text {symm }}}{\partial x}(x(s), y(s)) .
$$

Unlike the situation of the optimal management problem, the curve parameter $s$ has not an interpretation in terms of time; it is a purely auxiliary quantity. For the lake game, this yields

$$
x^{\prime}(s)=u-g(x), \quad y^{\prime}(s)=\rho y+2 c x+y g^{\prime}(x) .
$$

Since there is no initial condition, all integral curves of the system (20) that satisfy the transversality condition are candidates to generate Nash feedback equilibrium strategies.

Kossioris et al. (2008) report graphs generated by such families of integral curves as Nash feedback equilibria. However, these graphs are only defined on subintervals $U$ of the state space $X=[0, \infty)$. To be a Nash equilibrium, no deviation from the equilibrium strategy should generate a higher payoff. But as it is possible to construct a strategy that takes the state out of the interval $U$, the payoff for the players that play a strategy only defined in $U$ becomes undefined. To make such strategies admissible, the game has to be changed in such a way that no player can play an action taking the system out of $U$. But for the unrestricted game, these strategies cannot be admitted as solutions. Only those integral curves can constitute Nash equilibrium strategies that are defined on the whole state space.

For the lake game, the equations (20) coincide with the system (7)-(8); the optimal policy function given in Figure 6 is therefore also the Nash feedback strategy of a player in the game. However, as there are now several players, the resulting steady state will be lower. Figure 10 illustrates two situations.

Consider first Figure 10(a), where the lake is reversible, but close to fragile. Under cooperation, the joint action of the cooperators is equal to optimal management, as illustrated in Figure 6. As noted before, the oligotrophic steady state is close to the tipping point of the lake dynamics, and the strong negative feed- 


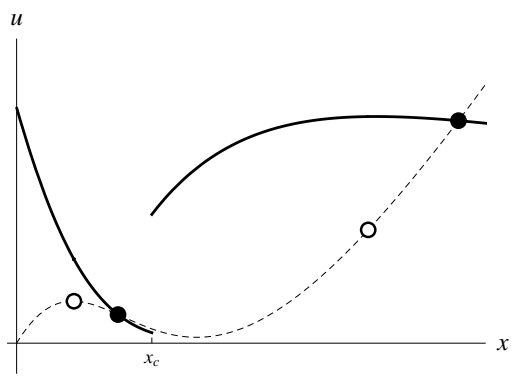

(a) $b=0.51, c=0.5$

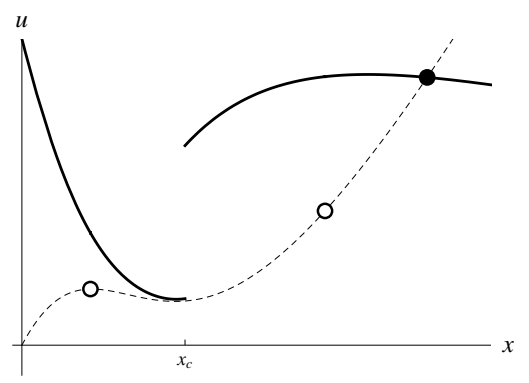

(b) $b=0.58, c=0.5$

Figure 10: Two-player symmetric Nash equilibrium feedback strategies for the lake game (solid), as well as the associated long-term steady state condition $2 u=g(x)$ (dashed). The circles indicate long-term steady states under cooperation (white) and noncooperation (black). Parameters: $n=2, \rho=0.03$.

back provided by the optimal policy function stabilises it. Under cooperation, each of the players are allowed half of the pollution level of the optimal pollution level; in Figure 10(a) this level is equal to the value of the vertical coordinate of the white circles.

The cooperative level is much lower than the pollution level of the noncooperative feedback strategy at the tipping point. On the other hand, noncooperation results in an oligotrophic steady state that is past the tipping point, the left black circle in Figure 10(a), where both the pollution level in the lake is higher, and the pollution stream allowance of the players is lower, than in the cooperative steady state. Moreover, the stabilising feedback is much weaker: the graph of the feedback strategy runs close to that of the steady state condition $u=g(x) / 2$ of the lake, indicating that time-relaxation towards the steady state will be slow.

The relative locations of the eutrophic steady state under cooperation and noncooperation show a trade-off: under noncooperation, a worse environment sets off higher production.

If the lake is more robust, as in Figure 10(b), the eventual outcome of the economic interactions deteriorates: under noncooperation, the oligotrophic steady state disappears, and instead there is a discontinuity in the strategies of the players at a critical state $x=x_{c}$. The low values of the pollution stream for state values lower than but close to the critical state imply that the lake will remain for 
a long time still at low pollution levels; then, when the critical state is crossed, the lake deteriorates rapidly towards the eutrophic steady state.

Apparently, if the lake is robust and therefore can sustain more pollution, the danger of an environmental regime shift is not sufficiently pressing for it to be prevented; it is the fragile lake that more easily survives, also because ending up in the eutrophic domain is much more costly in the long run.

\section{Taxes}

A possible way to alleviate the effects of the prisoner's dilemma in the shallow lake problem, or more generally in problems where different agents use a common pool resource, is to impose taxes that correct the shadow value of the stock. Mäler et al. (2003) and Kossioris et al. (2011) consider such tax schemes for the lake problem sketched above; Heijnen and Wagener (2013) model the pollution stream as an output of a capital intensive industry and they consider taxes for this situation. Heijdra and Heijnen (2013) show that in presence of hysteresis, a policy of finite duration can have lasting beneficial effects.

\subsection{Time-dependent tax rates in the lake problem}

A proportional tax $\tau=\tau(t)$ on the pollution stream, imposed on players using open-loop strategies, changes the total benefits of player $i$ to

$$
I_{i}=\int_{0}^{\infty} \mathrm{e}^{-\rho t}\left(\beta_{\mathrm{a}}\left(u_{i}\right)+\beta_{\mathrm{e}}(x)+\tau u_{i}\right) \mathrm{d} t .
$$

Given the pollution streams of the other players, the dynamic optimisation problem of player $i$ then requires maximising the Pontryagin function

$$
P=\beta_{\mathrm{a}}\left(u_{i}\right)+\beta_{\mathrm{e}}(x)+\tau u_{i}+y_{i}\left(\sum_{j=1}^{n} u_{j}-g(x)\right),
$$

which leads to

$$
\beta_{\mathrm{a}}^{\prime}\left(u_{i}\right)+\tau+y_{i}=0 .
$$


Let $u_{\mathrm{c}}(t)=\frac{1}{n} u_{\mathrm{o}}(t)$ be the optimal pollution stream allowance for each player under cooperation, which is the $n$ 'th fraction of the optimal pollution stream $u_{\mathrm{o}}$ of a single player. For $u_{i}=u_{\mathrm{c}}$, the corresponding shadow value of the lake for player $i$ equals

$$
y_{\mathrm{c}, i}=-\beta_{\mathrm{a}}\left(u_{\mathrm{c}}\right) .
$$

In order that the optimal choice of $u_{i}$ in an open-loop Nash equilibrium coincides with $u_{\mathrm{c}}$, it is necessary that

$$
\tau=y_{\mathrm{c}, i}-y_{i}
$$

"The tax bridges the gap between the social shadow cost of the accumulated phosphorus [...] and the private shadow cost of the accumulated phosphorus" (quoted from Mäler et al., 2003, p. 615). However, a time-varying tax rate is in practice difficult to implement. In Mäler et al. (2003), the authors therefore turn to a constant tax rate that changes the dynamics in such a way that the oligotrophic steady state coincides with the steady state under cooperation.

\subsection{State-dependent taxes in the lake problem}

Kossioris et al. (2011), in the situation that players use feedback strategies $u_{i}=$ $u_{i}(x)$, investigate the effect of state-dependent tax rates $\tau=\tau(x)$ given by low order polynomials: a constant rate is the simplest example in this class. Using a numerical algorithm to choose the tax rate optimally, they show that for a given initial value, a cubic state dependent tax rule can bridge almost two thirds of the gap between the payoffs per player in the noncooperative and the cooperative cases.

\subsection{Time-dependent taxes in a global warming model}

Models where an industry affects a natural resource, and which can sustain multiple equilibria, have been studied by Greiner and Semmler (2005), Greiner et al. (2010) and Janmaat (2012). The latter author considers the fish stock in a lake as productive capital; naturally, the state of the lake affects the capital stock. 
Greiner et al., slightly modifying the model of Greiner and Semmler, study global warming caused by the emission of greenhouse gases: mean atmospheric temperature $T$ and the concentration of greenhouse gases $M$, expressed as a multiple of the pre-industrial level, evolve according to

$$
\begin{aligned}
\dot{T} & =g(T)+\log M, \\
\dot{M} & =E-\mu M ;
\end{aligned}
$$

here $g$ is a nonlinear relation deriving from the earth's radiative energy balance, and $E$ are industry emissions, taken to be proportional to the ratio of capital $K$ to abatement activities $A$, or per capita capital $k$ to per capita abatement $a$ :

$$
E \propto \frac{K}{A}=\frac{k}{a}
$$

The labour supply $L$ is assumed to grow at a rate $n$. Expressing everything in per capita units, per capita output takes the form

$$
y=b k^{\alpha} D(T)
$$

the damage function $D$ is decreasing, taking the value 1 for the pre-industrial mean temperature $T_{0}$. Output is spent on consumption $c$, abatement $a$, replacement of old capital, and income tax and emission tax, at rates $\tau$ and $\tau_{E}$ respectively:

$$
\dot{k}=(1-\tau) y-c-a-\tau_{E} \frac{E}{L}-(\delta+n) k .
$$

Optimising total welfare

$$
I=\int_{0}^{\infty} \mathrm{e}^{-\rho t} L \log c \mathrm{~d} t
$$

they find, for a certain parameter combination, a surface of indifference threshold points in the three-dimensional state space (see Figure 11). There are two attracting steady states under optimal management, 'warm', denoted in red, and 'cool', denoted in blue: the warm steady state has both higher values of the mean 


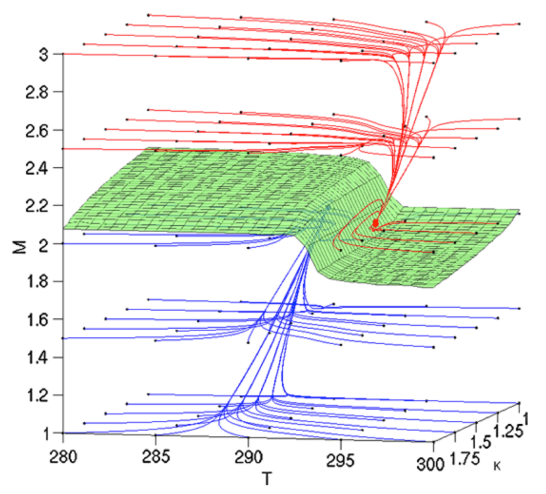

Figure 11: Indifference surface in the state space of the Greiner-GrüneSemmler model (after Greiner et al. (2010)).

temperature and of the steady state level of capital. For given values of $K$, the indifference thresholds are almost independent of $T$, except for a small interval around $T_{c} \approx 293$, where they decrease from $M \approx 2.1$ to $M \approx 1.8$.

Note the shape of the trajectories: for most initial points, first temperature is steered towards values around $T \approx 290$, that is about $17^{\circ} \mathrm{C}$, in the cool regime, or around $T \approx 296$, about $23^{\circ} \mathrm{C}$, in the warm regime. Only then significant changes to the capital and the pollution levels are effected by the optimal policy. In both situations, the asymptotic value of $M$ is about 2 , that is, twice the pre-industrial level of greenhous gases.

Greiner and Semmler (2005) discuss also a competitive economy, where the impact of the decisions of indiviual agents on the state of the environment is negligible. As in the situation of the lake problem, imposing the tax $\tau_{E}$ on emissions to correct the shadow value of the environment makes the agents internalises the negative externality.

\subsection{Constant tax rates in an extended lake problem}

Heijnen and Wagener (2013) extend the lake model by adding a capital-intensive industry with a fixed amount of labour and a variable amount of capital $k$; in the model, the state of the lake has no impact on the industry. As time-dependent taxes, as considered above Greiner and Semmler (2005); Mäler et al. (2003) are 
hard to implement in practice, they investigate how well constant tax rates can reduce the pollution externality.

In their model, industry per capita output $y=f(k)$ is spent on investment in new capital, consumption $\kappa$, or taxes, which in the model take the form of mandatory contributions to pollution abatement. Capital dynamics then take the form

$$
\dot{k}=f(k)-\kappa-(\delta+\tau \pi \eta) k ;
$$

here $\delta$ is the rate of depreciation of capital; $\eta$ the amount of pollutant per unit time generated by the use of a single unit of capital; $\pi$ the price of removing a unit of pollutant per unit time; and finally $\tau$ the imposed abatement level. The pollutant dynamics in the lake takes the form

$$
\dot{x}=(1-\tau \eta) k-g(x) .
$$

Two situations are compared: in the first, a social planner tries to maximise

$$
I=\int_{0}^{\infty}\left(\log \kappa-c x^{2}\right) \mathrm{e}^{-\rho t} \mathrm{~d} t
$$

by choosing the consumption level $\kappa$ optimally. The maximum principle then yields the following set of equations, after eliminating the shadow price of capital in terms of consumption:

$$
\begin{aligned}
\dot{\kappa} & =\left(f^{\prime}(k)-(\rho+\delta+\tau \pi \eta)\right) \kappa+(1-\tau) \eta q \kappa^{2}, \\
\dot{q} & =\left(\rho+g^{\prime}(x)\right)+2 c x ;
\end{aligned}
$$

here $q$ is the shadow value of the lake.

In the second 'competitive' situation, there is a continuum of identical consumers, supplying their labour to the industry at the prevailing wage rate $w=$ $w(t)$. Wages are either spent on consumption or put in a bank account at an interest rate $r=r(t)$, which, in turn, is determined by the marginal productivity 
of capital:

$$
f^{\prime}(k)=r(t)+\delta+\tau \pi \eta .
$$

The bank balances evolve as

$$
\dot{b}=r b-\kappa+w,
$$

subject to the condition that their discounted value is bounded away from $-\infty$; this is a 'No Ponzi' condition. As actions of each individual consumer have negligible effects on the total amount of pollution, every consumer maximises just discounted utility from individual consumption

$$
\int_{0}^{\infty} \log (\kappa) \mathrm{e}^{-\rho t} \mathrm{~d} t
$$

Applying the maximum principle to this dynamic optimisation problem, and expressing the costate variable in terms of the consumption yields eventually that

$$
\dot{\kappa}=(r(t)-\rho) \kappa .
$$

As the industry is perfectly competitive, the marginal productivity of capital equals the price of capital, that is

$$
r(t)=f^{\prime}(k)-\delta-\tau \pi \eta .
$$

This yields eventually

$$
\dot{\kappa}=\left(f^{\prime}(k)-(\rho+\delta+\tau \pi \eta)\right) \kappa .
$$

Comparing this with (24) shows that here the consumers do not take the state of the lake into account in their consumption decisions.

Heijnen and Wagener investigate a parameter configuration where, without abatement, the social planner keeps the lake in the oligotrophic state by the social planner, whereas it flips to the eutrophic state under competition. This is driven 
by consumption: while the social planner imposes low pollution streams, and implicitly low consumption levels, in the competitive case there is overconsumption and overpollution.

Increasing the abatement rate $\tau$ improves the social planner case somewhat: pollution abatement actually allows the industrial production to increase, as pollution effects are compensated for, which leads to higher consumption levels. When the abatement tax increases past a certain level, abatement becomes so costly that consumption starts to decrease again. However, the effects on the total welfare level are modest.

This is in sharp contrast to the competitive case: here the total welfare level increases quickly, though consumption decreases somewhat, until the lake no longer enters the eutrophic region. When that is the case, the welfare level of the competitive case is almost equal to that of the social planner case, and it follows the same pattern. Put differently, the external pollution costs can be largely avoided by imposing a tax whose proceeds are earmarked for abatement.

\section{Uncertain regime shifts}

The shallow lake system models a system that can exhibit a regime shift for which the dynamics are deterministic and fully known. This section will discuss a number of articles where a regime shift may occur with a given probability that may or may not depend on the actions of the agents. In the 1980s, Reed has considered regime shifts occurring with a natural hazard rate for resource extraction problems, more precisely for forests in the presence of fire risk (Reed, 1984) and the catastrophic collapse of fisheries (Reed, 1988). Clarke and Reed (1994) (see also Tsur and Zemel, 1998) extended this to hazard rates that depended on pollution concentration, and thus indirectly on the actions of the agents in the problem. They found that if the hazard rate of a regime shift increased sufficiently quickly with pollution, optimal pollution levels and consumption levels are lower than in the case where there is no possibility of a regime shift. If this kind of precautionary behaviour on the part of the agents is optimal, a 'precautionary principle' 
is said to hold. What is puzzling about these results, however, is that in some situations, the optimal behaviour of agents is ambiguous. That is, even in the presence of pollution-induced risk of regime shifts, it may be optimal to consume more, rather than less, than in the situation without risk.

To understand the underlying mechanisms, consider the following optimal harvesting problem discussed by Polasky et al. (2011). A manager is to maximise discounted revenues from harvesting

$$
I=\int_{0}^{\infty} \mathrm{e}^{-\rho t} p u \mathrm{~d} t
$$

with $p$ the unit price of the harvested good, subject to stock dynamics

$$
\dot{x}=G(x)-u,
$$

as well as the requirements that $x \geq 0, u \geq 0$ for all $t$. There is a (stochastic) time $\tau$ such that for $0 \leq t \leq \tau$, stock growth is given by $G(x)=G_{1}(x)$, whereas for $t>\tau$, the stock dynamics satisfy $G(x)=G_{2}(x)$. It is possible that the regime shift from $G_{1}$ to $G_{2}$ takes never place. Both functions are strictly concave, take a maximum for some positive stock value, and satisfy $G_{i}(0)=0(i=1,2)$. Deterioration of the system after the regime shift is expressed by the assumptions that $G_{1}(x) \geq G_{2}(x)$ and $G_{1}^{\prime}(x) \geq G_{2}^{\prime}(x)$ for all $x \geq 0$.

The optimisation problem is most conveniently stated and solved in terms of two Hamilton-Jacobi-Bellman equations: the first for the value $V_{1}$ of the stock before the shift, and the second for the value $V_{2}$ after the shift. The solution is sketched for the, simpler, second case, after which the Hamilton-Jacobi-Bellman equation and the result for the first case are stated.

After the regime shift, the natural growth function of the stock is $G_{2}(x)$. The Hamilton-Jacobi-Bellman equation for $V_{2}$ reads as

$$
\rho V_{2}(x)=\max _{u \geq 0}\left\{p u+V_{2}^{\prime}(x)\left(G_{2}(x)-u\right)\right\} .
$$

As the integrand of the revenue $I$ is linear in the harvest rate $u$, maximising over $u$ 
results in a so-called bang-bang harvesting policy:

$$
u= \begin{cases}0 & \text { if } V_{2}^{\prime}(x)>p \\ \text { indeterminate } & \text { if } V_{2}^{\prime}(x)=p \\ \infty & \text { if } V_{2}^{\prime}(x)<p\end{cases}
$$

That is, the stock grows at the natural rate as long as its shadow value is above the market price for the harvest; if it is below the market price, it is harvested at the maximal rate.

The solution of equation (29) is

$$
V_{2}(x)= \begin{cases}\mathrm{e}^{-\rho \theta(x) \frac{p G_{2}\left(x_{2}\right)}{\rho}} & \text { for } 0 \leq x \leq x_{2}, \\ p\left(x-x_{2}\right)+\frac{p G_{2}\left(x_{2}\right)}{\rho} & \text { for } x>x_{2}\end{cases}
$$

here $x_{2}$ is the unique solution of the 'golden rule'

$$
G_{2}^{\prime}\left(x_{2}\right)=\rho,
$$

and $\theta(x)$ is the time needed by the stock to reach the equilibrium level $x_{2}$, starting from the initial level $x$. That is, when starting below $x_{2}$, the optimal harvesting policy does not harvest until the stock level reaches $x_{2}$, after which it harvests at the equilibrium rate $G_{2}\left(x_{2}\right)$. When the initial stock is larger than $x_{2}$, the excess stock $x-x_{2}$ is harvested and sold instantly, after which harvest proceeds as before at the equilibrium rate.

Consider now the situation before the regime shift. Recall that $\tau$ denotes the stochastic time at which the shift occurs. The probability that the shift occurs in a time interval $[t, t+h)$, conditional on the fact that it did not occur before time $t$, satisfies

$$
\lim _{h \rightarrow 0} \frac{P(\tau \in[t, t+h) \mid \tau \geq t)}{h}=\lambda(x(t)),
$$

where the limit $\lambda(x)$ is the 'hazard rate' at state $x$. The Hamilton-Jacobi-Bellman 
equation for the stock value in the first regime is then of the form

$$
\rho V_{1}(x)=\max _{u \geq 0}\left\{p u+V_{1}^{\prime}\left(G_{1}(x)-u\right)+\lambda(x)\left(V_{2}(x)-V_{1}(x)\right)\right\} .
$$

If the rate of stock growth deteriorates after the shift, it follows that $V_{2}(x) \leq$ $V_{1}(x)$; the last term in the equation then models the penalty incurred if the regime shift occurs.

The golden rule for the situation before the shift, which is analogous to condition (31) for the steady state after the shift, states that a steady state $x_{1}$ under optimal harvesting satisfies

$$
G_{1}^{\prime}\left(x_{1}\right)=\rho+\lambda\left(x_{1}\right)\left(1-\frac{V_{2}^{\prime}\left(x_{1}\right)}{p}\right)+\frac{\lambda^{\prime}\left(x_{1}\right)}{\rho+\lambda\left(x_{1}\right)}\left(G_{1}\left(x_{1}\right)-\frac{\rho V_{2}\left(x_{1}\right)}{p}\right) .
$$

This equation furnishes information both if the shift probability is independent of the stock level (exogenous shift) or dependent (endogenous shift), and both if the stock collapses after the shift $\left(V_{2}(x)=0\right.$ for all $\left.x\right)$, or if only the growth dynamics changes. There are four combinations in total.

First, consider the exogenous shifts, for which $\lambda$ is constant. With stock collapse, equation (32) reads as

$$
G_{1}^{\prime}\left(x_{1}\right)=\rho+\lambda
$$

As $G_{1}^{\prime}$ is a decreasing function, it follows that the steady state $x_{1}$ decreases relative to the situation without the possibility of a regime shift: the optimal harvest rate increases, as the expected time interval over which harvesting is possible decreases: the planner is more impatient.

If however only the growth dynamics deteriorates, the steady state $x_{2}$ after the shift is lower than $x_{1}$, and the excess stock is harvested immediately. This implies that the second term on the right hand side of equation (32) vanishes. The third term vanishes as $\lambda^{\prime}(x)=0$ for a constant hazard rate, and the equation takes the form

$$
G_{1}^{\prime}\left(x_{1}\right)=\rho
$$


In this situation, the steady state under optimal harvesting is independent on the natural hazard rate.

Endogenous shifts with total stock collapse lead to

$$
G_{1}^{\prime}\left(x_{1}\right)=\rho+\lambda\left(x_{1}\right)+\frac{\lambda^{\prime}\left(x_{1}\right)}{\rho+\lambda\left(x_{1}\right)} G_{1}\left(x_{1}\right) .
$$

Viewing the stock level as environmental quality, the hazard rate is expected to decrease as the stock level increases. If the decrease is sufficiently rapid, the result of Clarke and Reed is recovered that the right hand side of (33) is smaller than $\rho$, and the steady state value $x_{1}$ is larger than in the case without risk of collapse. On the other hand, for marginal hazard rates that are small in absolute value, impatience of the planner leads to a decrease of the steady state stock, much like in the case of exogenous risk of stock collapse.

Finally, for endogenous shifts with deteriorating growth dynamics, and for decreasing hazard rates, the last term on the right hand side of (32) is negative; this involves some reasoning. As the second term in the expression is again equal to 0 , it follows that here the steady state stock is always greater than in the situation without regime shifts. Put differently: if the hazard rate decreases with the stock, and if the planner does not lose stock at the moment of collapse, the optimal harvesting rate is precautionary compared with the situation without risk of collapse.

\section{Conclusion}

Negative feedbacks stabilise regimes; positive feedbacks differentiate between regimes. Natural systems under stress can have several regimes; if the stresses are too large, a regime may lose stability and the system shifts to a different regime (Figure 1). Management improves the robustness of systems by strengthening the negative feedback: the oligotrophic steady state of Figure 6 and the steady state of Figure $7(\mathrm{a})$, both marginally stable under constant loading, are robustly stable under optimal management. 
If the use of the natural system is shared between agents, the situation deteriorates, as is usual with common pool problems. There are generally various situations, depending on the precise specifications of the system, classified for the quasi-static situation in the bifurcation diagram of Figure 9. In the prototypical lake system, there is a large parameter region for which there are either two candidate steady state outcomes. Though for most of this region, the oligotrophic steady state maximises the player's welfare, only for a small subregion this steady state is the unique outcome of a Nash equilibrium in loading strategies. The other possibilities are that it is a welfare-preferred outcome of two Nash equilibria, or that it is dominated by a Nash equilibrium resulting in the eutrophic steady state. A final possibility, which is uncommon and which derives from the fact that this game is dependent on initial states, is that the welfare-preferred steady state is not reachable if the initial state is outside a certain region.

In the situation with discounted future benefit streams, the whole time evolution determines the resulting outcome, not only the steady states. Modelling the behaviour of the agents in terms of strategies, taken from certain strategy classes, tax rules can be devised that sustain the cooperative outcome. This may result in vastly better long-term economic performance of the system (Figure 10(b)).

All this analysis presupposes knowledge of the response of the natural system. If the occurrence of a regime shift is uncertain, but the actions of the agents influence the probability of the shift occurring, one strand of thought advises to increase consumption, implicitly stressing the environment, in order to make optimal use of the time before the collapse - "Après nous, le déluge". The precautionary principle, which advises to reduce stress on the environment in order to retard the moment of collapse, embodies the opposite stance. It turns out that, depending on particulars, both situations may be optimal if the collapse of the environmental system also entails the collapse of the natural resources sustained by the system. If there is however only a regime shift of the system, but no instantaneous deterioration of the stock, then precautionary behaviour is unambiguously to be preferred.

The analysis of uncertain regime shifts suggests that it may be of interest to 
consider learning models in the future: as the system moves to - 'explores' regions of the state space not visited previously, the agents learn about the dynamics there, and modify their behaviour accordingly. Also, the assumptions of fully rational behaviour of agents might have to be relaxed. Finally, the institutional problem remains challenging: how to decentralise the decision problem such that the negative externalities from environmental degradation are, at least partly, internalised (cf. Starrett, 1972), and how to do this in a practicable way.

\section{References}

Brock, W.A. and Starrett, D. (2003). Nonconvexities in ecological managment problems. Environmental and Resource Economics, 26(4), 575-624.

Case, J.H. (1979). Economics and the competitive process. New York University Press, New York.

Clarke, H.R. and Reed, W.J. (1994). Consumption/pollution tradeoffs in an environment vulnerable to pollution-related catastrophic collapse. Journal of Economic Dynamics and Control, 18(5), 991-1010.

Crépin, A.S., Biggs, R., Polasky, S., Troell, M., and de Zeeuw, A. (2012). Regime shifts and management. Ecological Economics, 84, 15-22.

Dasgupta, P. and Mäler, K.-G. (ed.) (2004). The economics of non-convex ecosystems. Kluwer.

Dechert, W.D. and Nishimura, K. (1983). A complete characterization of optimal growth paths in an aggregated model with a non-concave production function. fournal of Economic Theory, 31, 332-354.

Dockner, E.J. and Van Long, N. (1994). International pollution control: cooperative versus non-cooperative strategies. Journal of Environmental Economics and Management, 24, 13-29. 
Dockner, E.J. and Wagener, F.O.O. (2013). Markov-perfect Nash Equilibria in Models With a Single Capital Stock. Working paper 13-03, CeNDEF, University of Amsterdam.

Greiner, A., Grüne, L., and Semmler, W. (2010). Growth and climate change: threshold and multiple equilibria. In Dynamic systems, economic growth, and the environment (ed. J. Cuaresma, T. Palokangas, and A. Tarasyev), Volume 12, Dynamic modeling and econometrics in economics and finance, pp. 63-78. Springer.

Greiner, A. and Semmler, W. (2005). Economic growth and global warming: A model of multiple equilibria and thresholds. Fournal of Economic Behavior \& Organization, 57, 430-447.

Hardin, G. (1968, December). The tragedy of the commons. Science, 162(3859), $1243-1248$

Heijdra, B.J. and Heijnen, P. (2013). Environmental abatement and the macroeconomy in the presence of ecological thresholds. Environmental and Resource Economics, 55(1), 47-70.

Heijnen, P and Wagener, FOO (2013). Avoiding an ecological regime shift is sound economic policy. Journal of Economic Dynamics and Control, 37(7), 1322-1341.

Janmaat, J.A. (2012). Fishing in a Shallow Lake: Exploring a Classic Fishery Model in a Habitat with Shallow Lake Dynamics. Environmental \& Resource Economics, 51, 215-239.

Kiseleva, T. and Wagener, F.O.O. (2010). Bifurcations of one-dimensional optimal vector fields in the shallow lake system. Fournal of Economic Dynamics \& Control, 34, 825-843.

Kiseleva, T. and Wagener, F.O.O. (2011). Bifurcations of optimal vector fields. CeNDEF Working paper 11-05, University of Amsterdam. 
Kossioris, G., Plexousakis, M., Xepapadeas, A., and de Zeeuw, A. (2011). On the optimal taxation of common-pool resources. Journal of Economic Dynamics and Control, 35, 1868-1879.

Kossioris, G., Plexousakis, M., Xepapadeas, A., de Zeeuw, A., and Mäler, K.-G. (2008). Feedback Nash equilibria for non-linear differential games in pollution control. Journal of Economic Dynamics and Control, 32, 1312-1331.

Krugman, P. (1991). Increasing returns and economic geography. The fournal of Political Economy, 99(3), 483-499.

Majumdar, M. and Mitra, T. (1982). Intertemporal allocation with a non-convex technology: The aggregative framework. fournal of Economic Theory, 27(1), 101-136.

Mäler, K.-G., Xepapadeas, A., and de Zeeuw, A. (2003). The economics of shallow lakes. Environmental and Resource Economics, 26(4), 105-126.

Polasky, S., de Zeeuw, A., and Wagener, F.O.O. (2011). Optimal management with potential regime shifts. Fournal of Environmental Economics and Management, 62, 229-240.

Reed, W.J. (1984). The effects of the risk of fire on the optimal rotation of a forest. Journal of Environmental Economics and Management, 11(2), 180-190.

Reed, W.J. (1988). Optimal harvesting of a fishery subject to random catastrophic collapse. IMA fournal of Mathematics Applied in Medicine \& Biology, 5(3), 215235.

Rincón-Zapatero, J.P., Martínez, J., and Martín-Herrán, G. (1998). New method to characterize subgame perfect Nash equilibria in differential games. Journal of Optimization Theory and Applications, 96(2), 377-395.

Romer, P.M. (1986). Increasing returns and long-run growth. The fournal of Political Economy, 94(5), 1002-1037. 
Scheffer, M. (1998). Ecology of shallow lakes. Chapman \& Hall, London.

Seierstad, A. and Sydsaeter, K. (1987). Optimal control with economic applications. North-Holland, Amsterdam.

Sethi, S.P. (1977). Nearest feasible paths in optimal control problems: Theory, examples, and counterexamples. Journal of Optimization Theory and Applications, 23(4), 563-579.

Skiba, A.K. (1978). Optimal growth with a convex-concave production function. Econometrica, 46, 527-539.

Starrett, D.A. (1972). Fundamental nonconvexities in the theory of externalities. Journal of Economic Theory, 4(2), 180-199.

Tahvonen, O. and Salo, S. (1996). Nonconvexities in optimal pollution accumulation. fournal of Environmental Economics and Management, 31(2), 160-177.

Treadway, A.B. (1969). On rational entrepreneurial behaviour and the demand for investment. The Review of Economic Studies, 36(2), 227-239.

Tsur, Y. and Zemel, A. (1998). Pollution control in an uncertain environment. Journal of Economic Dynamics and Control, 22, 967-975.

Tsutsui, S. and Mino, K. (1990). Nonlinear strategies in dynamic duopolistic competition with sticky prices. Journal of Economic Theory, 52, 136-161.

von Weizsäcker, C. (1965). Existence of optimal programs of accumulation for an infinite time horizon. Review of Economic Studies, 32, 85-104.

Wagener, F.O.O. (2003). Skiba points and heteroclinic bifurcations, with applications to the shallow lake system. Fournal of Economic Dynamics and Control, 27, $1533-1561$.

Wirl, F. (1996). Dynamic voluntary provision of public goods: Extension to nonlinear strategies. European fournal of Political Economy, 12, 555-560. 